\title{
Multimodal Data Analysis and Visualization to Study the Usage of Electronic Health Records
}

\author{
Nadir Weibel $^{1}$, Shazia Ashfaq ${ }^{2}$, Alan Calvitti ${ }^{2}$, James D. Hollan ${ }^{1}$, Zia Agha ${ }^{2}$ \\ ${ }^{1}$ University of California San Diego, La Jolla CA 92093, USA \\ ${ }^{2}$ HSRD VA San Diego Healthcare System, San Diego CA 92161, USA \\ \{weibel, hollan\}@ucsd.edu, \{shazia.ashfaq, alan.calvitti, zia.agha\}@va.gov
}

\begin{abstract}
Understanding interaction with Electronic Health Records (EHR), often means to understand the multimodal nature of the physician-patient interaction, as well as the interaction with other materials (e.g. paper charts), in addition to analyze the tasks fulfilled by the doctor on his computerized system. Recent approaches started to analyze and quantify speech, gaze, body movements, etc. and represent a very promising way to complement classic software usability. However, it is hard to characterize multimodal activity, since often it requires manual coding of hours of video data. We present our approach to use automatic tracking of body, audio signals and gaze in the medical office to achieve multimodal analysis of EHR.
\end{abstract}

\section{INTRODUCTION}

The US Agency for Healthcare Research and Quality (AHRQ) and the National Institute of Standards and Technology (NIST) have prioritized the need to measure and improve Electronic Health Record (EHR). AHRQ has emphasized research on: "documenting patterns of clinician information use in EHR systems" and "developing an understanding of, and ways to measure, the impact of usability and information design on efficiency of care delivery." [1]

Current EHR user interfaces are based on the windowing model and burdensome pull-down/popup menus, with limited information retrieval and business-rule logic automation. The result is excessive time spent navigating nested menus, cuttingpasting text or lab results across screens, and searching for specific data from a sea of boilerplate text. Pressed for time, physicians multitask between using EHRs and communicating with patients and may defer some EHR data entry. This creates risk for errors and ineffective communication with patients.

Another key aspect of clinical workflow is documentation, which consumes much time in clinical work, even more than direct patient care. Some authors have argued that although relevant categories of medical records are defined in the healthcare IT systems, "the practices through which the document is written, read and used within consultation have been largely ignored." In summary, EHR systems are inefficient, introduce human-computer interaction problems, increase cognitive load, and alter the dynamic of clinical activities [2]-[6].

\section{Patient-Centered Evaluation of EHR}

We are conducting two research studies called PACE (Patient Centered Evaluation of EHR) and QUICK (Quantifying EHR Usability to Improve Clinical Work). These studies are motivated by the current knowledge gaps in profiling EHR use in clinical settings and understanding consequences for communication and workflow. The studies take an interdisciplinary approach, borrowing methods and knowledge from cognitive science, HCI, medical informatics and health services research. The studies aims are: (1) to develop process-level quantitative measures of EHR usage, clinical workflow, and provider-patient communication; (2) to develop indicators of EHR usability and workflow, from the quantitative measures of EHR usage; (3) to use data on EHR usage patterns, clinical work, and communication to develop composite models of EHR use and clinical workflow; and (4) to explore associations between EHR use and workflow. We compare different clinical environments and care services such as whether EHR use, clinical workflow, or communication patterns change between sites, specialties, or EHR platforms.

\section{FROM FORMATIVE TO REAL-WORLD EVALUATION}

Traditional usability studies consist of formative or summative evaluations carried out in laboratory settings with test patients for the sake of facilitating comparisons. This approach treats the EHR as a standalone system that can be evaluated in controlled environments. While this approach is suitable for evaluating specific functions, it is not informative of EHR usage in real clinical settings Our approach stresses sampling variability from real-world clinical settings.

To systematically study physician-patient-EHR interaction patterns while preserving the sequence and timing of the building block events we measure: EHR activity (based on mouse plus keyboard activity), clinicians nonverbal clinical workflow, and patient-provider verbal communication patterns. In addition we collect physician and patient satisfaction measures, patient-provider agreement, and physician cognitive load.

While some of the data collected from the EHR use can be automatized (i.e. by using software to track mouse, key presses, active windows, etc., such as Morae $^{1}$ ), clinical workflow (e.g. gazes to patient, caregiver, computer, paper records, physical exam, interaction with second provider, talking with patient, patient talking with physician) is captured by video and audio recording of the visit via an unobtrusive video camera and must be manually coded and timestamped.

By mining EHR data, verbal/non-verbal clinical communication, either individually or jointly, a range of quantitative profiles of EHR usability, provider-patient communication, and clinical workflow can be developed. Analysis of these profiles along the visit timeline enables to study complex clinical workflow processes [7]. We use composite visualizations adapting chromogram plots [8] that make apparent the detailed sequence of activities to show common patterns, clusters, variation, and task switching (e.g. within the EMR Tabs, such as Notes, Order, Medications). This enables objective measuring and comparison of EHR activity at multiple levels of aggregation. Fig. 1 shows the result of one of our studies in this context.

\footnotetext{
${ }^{1}$ http://www.techsmith.com/morae.html
} 


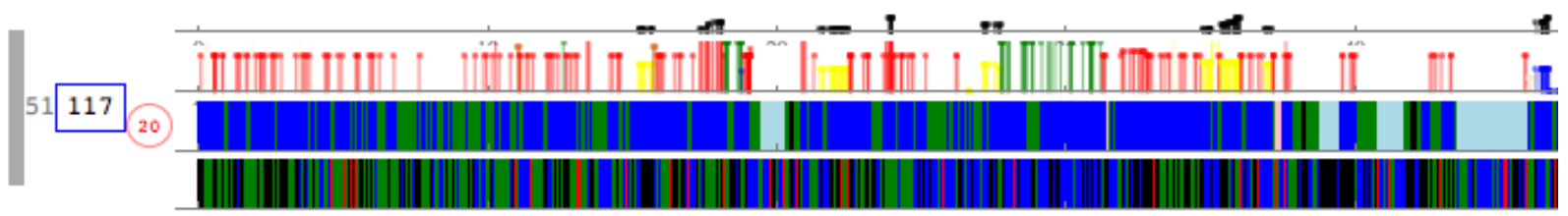

Fig. 1. Chromogram displaying along a timeline measures for a single outpatient visit (60 minutes). The four rows of events correspond to (top to bottom): EHR mouse activity for the order entry, mouse activity color-coded to top-level screen; physician nonverbal clinical workflow activity; patient-physician vocalizations.

\section{Do We NeED THE MANuAl CODER?}

While multimodal analysis of EHR interaction and usability is extremely interesting and opens up new avenues to really understand the complex interaction in the medical office and with the EHR, the process of coding the recorded data is extremely tedious. In our studies research assistants spent numerous hours to code the data recorded during office visits.

Instead, we propose to use the cutting-edge, but extremely available and affordable technology integrated in the Microsoft Kinect for Windows. ${ }^{2}$ In addition to normal video data, the Kinect enables continuous recording of depth information and is able to track the source angle of an audio signal. By leveraging those sensors it is possible to automatically detect body joints and involved participant, and use the recorded information to generate the previously manually coded data on clinical verbal and non-verbal communication and interaction, this time in an automatic or semi-automatic way.

We built an application that can be used to easily collect, and automatically generate and visualize a synchronized collection of multiple data stream. We use ChronoViz [9] to process the collected data and our new data collection application Kinect4ChronoViz to capture it. Figure 2 shows an example of data collected with a Kinect in a medical office using our tool and and analyzed in ChronoViz. After placing the Kinect(s) in the room, our tool enables researchers to easily collect data by pressing a single button. Data is bundled and synchronized and automatically presented in ChronoViz.

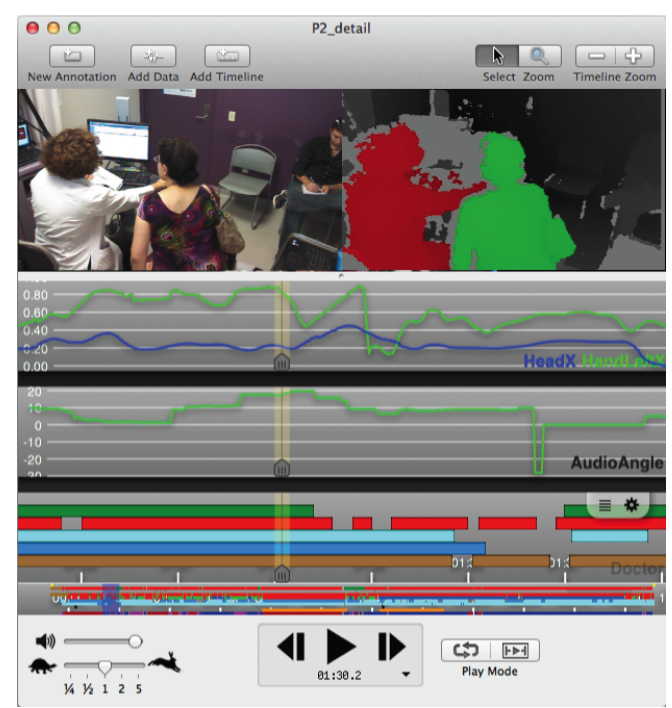

Fig. 2. ChronoViz shows data collected during a medical consultation through Kinect4ChronoViz. Top: RGB and depth data from the Kinect; Middle: position of some of the joints, and direction of the source audio data; Bottom: manual annotations. Minutes after the data has been collected, movement of the body (e.g. positions of hands and head), or speech turn-taking (indicated by the "Audio Angle" timeline), are visualized in ChronoViz.

\footnotetext{
${ }^{2} \mathrm{http}: / /$ kinectforwindows.org
}

We are currently developing plugins that automatically segment the data based on predefined parameters (e.g. identify when the physician or the patient is talking, based on their position with respect to the Kinect). Our preliminary results show that the developed methodology is able to process the activity that mostly relates to EHR, as well as physicianpatient interactions such as gestural and verbal interactions. Leveraging this automatic and semi-automatic classification has the potential to enable investigations of EHR interactions to larger scales, and inform the design of new EHR interfaces.

\section{CONClusion And Future Work}

While we are just starting to use automatic tracking of verbal and non-verbal communication and interaction in the medical office, we plan to validate the accuracy of our technique using manual coders as ground truth. We are also already integrating in our approach additional techniques that will allow us to track gaze as well as head positioning, and therefore measure visual attention (to the EHR, to the patient). All in all, we see an incredible opportunity to use tools such as Kinect4ChronoViz to automatically annotate hours of interaction with the EHR (and not only). This approach will enable researchers to reduce of a few orders of magnitude the problem of manual coding, and open up possibilities to analyze much wider datasets, in many different domains. This will ultimately enable researchers to gain better understanding of the multimodal nature of interactions. Our goal is to empower researchers with novel analysis methods that will lead to increased efficacy of EHR and ultimately improved healthcare.

\section{REFERENCES}

[1] Agency for Healthcare Research and Quality, "Electronic health record usability: evaluation and use case framework," 2009.

[2] C. Heath and P. Luff, "Documents and professional practice: bad organizational reasons for good clinical records," in Proc. CSCW '96, 1996, pp. $354-363$.

[3] A. Shachak and S. Reis, "The impact of electronic medical records on patient-doctor communication during consultation: a narrative literature review," Journal of evaluation in clinical practice, vol. 15, no. 4, pp. 641-649, 2009.

[4] R. Hillestad, J. Bigelow, and A. Bower, "Can electronic medical record systems transform health care? potential health benefits, savings, and costs," Health Aff (Millwood), vol. 24(5), pp. 1103-17, 2005.

[5] A. K. Jha, C. M. DesRoches, E. G. Campbell et al., "Use of electronic health records in us hospitals," New England Journal of Medicine, vol. 360, no. 16, pp. 1628-1638, 2009.

[6] R. Koppel, J. Metlay, and A. Cohen, "Role of computerized physician order entry systems in facilitating medication errors," JAMA, vol. 293(10), pp. 1197-203, 2005.

[7] K. Zheng, R. Padman, M. P. Johnson, and H. S. Diamond, "An interfacedriven analysis of user interactions with an electronic health records system," JAMIA, vol. 16, no. 2, pp. 228-237, 2009.

[8] M. Wattenberg, F. Viégas, and K. Hollenbach, "Visualizing activity on wikipedia with chromograms," Proc. Interact '07, pp. 272-287, 2007.

[9] A. Fouse, N. Weibel, E. Hutchins, and J. D. Hollan, "ChronoViz: a system for supporting navigation of time-coded data," in Proc. CHI '11, 2011. 